\title{
Elliptical concentrators
}

\author{
Angel Garcia-Botella, Antonio Alvarez Fernandez-Balbuena, and Eusebio Bernabeu
}

\begin{abstract}
Nonimaging optics is a field devoted to the design of optical components for applications such as solar concentration or illumination. In this field, many different techniques have been used to produce optical devices, including the use of reflective and refractive components or inverse engineering techniques. However, many of these optical components are based on translational symmetries, rotational symmetries, or free-form surfaces. We study a new family of nonimaging concentrators called elliptical concentrators. This new family of concentrators provides new capabilities and can have different configurations, either homofocal or nonhomofocal. Translational and rotational concentrators can be considered as particular cases of elliptical concentrators. (C) 2006 Optical Society of America
\end{abstract}

OCIS codes: $\quad 350.4600,220.1770,350.6050$.

\section{Introduction}

Nonimaging optics is a field devoted to the design of components such as solar concentrators or illumination devices, where collecting systems are used instead of the usual image formation systems. This field was born from the work of Hintenberger and Winston ${ }^{1}$ and Winston. ${ }^{2}$ Several powerful new techniques have been developed for the design of reflective and refractive optical components, for instance, the flow line method and the Poisson bracket method. ${ }^{3}$ Some of the components developed with the aid of these techniques today are well-known products in the nonimaging optics industry. As a classical example, we can refer to the compound parabolic concentrator (CPC).

Many efforts have been made to improve the behavior of these components, to achieve the maximum theoretical concentration ratio, ${ }^{4}$ or to adapt the concentrator to several interesting target geometries. ${ }^{5}$ But many of these components have been designed with translational symmetries, rotational symmetries, or free-form surfaces. ${ }^{6}$ In this paper, we study concentrators with quadrilateral symme-

A. Garcia-Botella (angel.garciab@upm.es) is with the Departamento Física Aplicada a los Recursos Naturales, Universidad Politécnica de Madrid, Escuela Técnica Superior de Ingenieros de Montes, Ciudad Universitaria s/n, Madrid 28040, Spain. A. A. Fernandez-Balbuena and E. Bernabeu are with the Departamento de Óptica, Facultad Ciencias Físicas, Universidad Complutense de Madrid, Ciudad Universitaria s/n, Madrid 28040, Spain.

Received 28 February 2006; revised 26 May 2006; accepted 26 May 2006; posted 26 May 2006 (Doc. ID 68555).

0003-6935/06/297622-06\$15.00/0

(C) 2006 Optical Society of America try and a monotonically varying elliptical cross section in homofocal and nonhomofocal configurations. We call this type of symmetry elliptic cylindrical symmetry. These new elliptical concentrators (ECs) have interesting properties, such as two principal acceptance angles or new expressions for the invariants. Translational and rotational concentrators can be considered as particular cases of these new components.

This work is organized as follows: In Section 2 we present a theoretical background, focused on the study of the concentration ratio and the skew invariant for this new family of concentrators. In Section 3 we study some applications of ECs, not only as a concentrator but also as an optical component in illuminators. Section 4 presents numerical results for elliptical CPCs, using ray-tracing simulations. Finally, in Section 5, conclusions are given.

\section{Theoretical Background}

To study the properties of these concentrators, we apply the etendue invariant through optical systems without losses ${ }^{3}$ :

$$
n^{2} \mathrm{~d} x \mathrm{~d} y \mathrm{~d} L \mathrm{~d} M=n^{\prime 2} \mathrm{~d} x^{\prime} \mathrm{d} y^{\prime} \mathrm{d} L^{\prime} \mathrm{d} M^{\prime},
$$

where $n$ and $n^{\prime}$ are the refractive indices of the input and output media, $\mathrm{d} x \mathrm{~d} y$ and $\mathrm{d} x^{\prime} \mathrm{d} y^{\prime}$ are the input and output areas of the beam, and $\mathrm{d} L \mathrm{~d} M$ and $\mathrm{d} L^{\prime} \mathrm{d} M^{\prime}$ are the input and output angular extents. By integration of Eq. (1) for elliptical input and output surface apertures, and considering the general case with two different input and output angles, we obtain the following expression: 
$n^{2} 4 \pi a b \sin \theta_{a} \sin \theta_{b}=n^{\prime 2} 4 \pi a^{\prime} b^{\prime} \sin \theta_{a}{ }^{\prime} \sin \theta_{b}{ }^{\prime}$,

where $a$ and $b$ are the major and minor axes of the entrance aperture, $a^{\prime}$ and $b^{\prime}$ are the major and minor axes of the exit aperture, $\theta_{a}$ and $\theta_{a}{ }^{\prime}$ are the longitudinal acceptance and output angles in the direction of the major axes, and $\theta_{b}$ and $\theta_{b}{ }^{\prime}$ are the transversal acceptance and output angles in the direction of the minor axes. From this, we can evaluate the theoretical maximum concentration ratio for these optical components, in which all output angles equal $\pi / 2$ :

$$
C_{\max }=\frac{A}{A^{\prime}}=\frac{\pi a b}{\pi a^{\prime} b^{\prime}}=\left(\frac{n^{\prime}}{n}\right)^{2} \frac{1}{\sin \theta_{a}} \frac{1}{\sin \theta_{b}} .
$$

This equation shows a new family of concentrators that have two principal acceptance angles in the longitudinal and transversal directions. From Eq. (3), we can deduce two interesting and well-known particular cases. In the first case, $\theta_{b}=\theta_{a}$, Eq. (3) provides the theoretical maximum concentration ratio for axisymmetric optical components. The second case corresponds to $\theta_{a}=\pi / 2$. The expression then gives the theoretical maximum concentration ratio for linear concentrators. In other cases, taking $\pi / 2>\theta_{a}>\theta_{b}$, we always have

$$
\left(\frac{n^{\prime}}{n}\right)^{2} \frac{1}{\sin \theta_{b}}<\left(\frac{n^{\prime}}{n}\right)^{2} \frac{1}{\sin \theta_{a}} \frac{1}{\sin \theta_{b}}<\left(\frac{n^{\prime}}{n \sin \theta_{b}}\right)^{2},
$$

which means that the EC has a smaller concentration ratio than an axisymmetric concentrator and a greater concentration ratio than a linear concentrator.

It is possible to study this new family of concentrators using a particular orthogonal coordinate system, called the elliptic cylindrical coordinate system, $(u, v, z)$ (Fig. 1). This coordinate system has the following conversion equations:

$$
\begin{aligned}
& x=c \cosh (u) \cos (v), \\
& y=c \sinh (u) \sin (v), \\
& z=z,
\end{aligned}
$$

where it is clear that the elliptic cylindrical coordinate system depends on the $c$ parameter. We can see that when $c \rightarrow 0$, the elliptic cylindrical coordinate system becomes a standard cylindrical coordinate system. On the other hand, when $c \rightarrow \infty$, the elliptic cylindrical coordinate system becomes a standard Cartesian coordinate system. This behavior allows us to consider the ECs as complementary concentrators between translational and rotational ones. Then, Eq. (3) can be considered as a general expression for the theoretical maximum concentration ratio.

An important question to study for these new components is the symmetry of the EC in an elliptic cylindrical coordinate system. We can say that the ECs

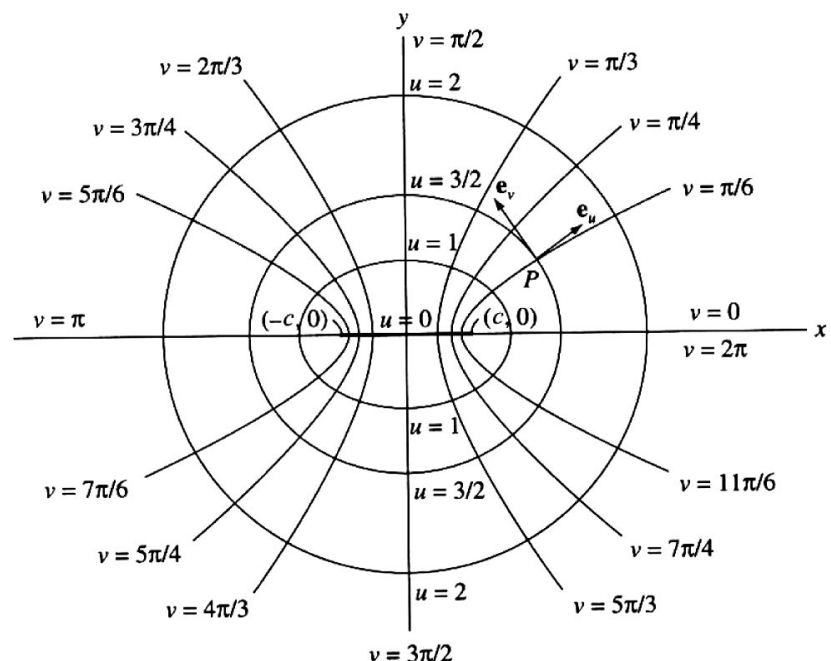

Fig. 1. Elliptic cylindrical coordinate system $(u, v, z)$ with focal length $c$ used to build ECs.

have rotational symmetry in the elliptic cylindrical coordinate system in the sense that, moving across the $v$ coordinate, which is an angular coordinate $(0 \leq v \leq 2 \pi)$, the concentrator geometry remains unchanged, ${ }^{7}$ in the same way that axisymmetric concentrators have rotational symmetry in a cylindrical coordinate system. The main consequence of this symmetry, by Hamiltonian optics, is that there exists an invariant for each ray propagating through the concentrator. For rotational symmetries, this invariant is the rotational skew invariant $h .^{3}$ Then we can write

$$
h=u_{\min } k_{v}
$$

where we have replaced the cylindrical radius $r_{\min }$ by the elliptical radius $u_{\min }$, and $k_{v}$ is the component of $\mathbf{k}$ in the tangential direction $\mathbf{v}$, with $\mathbf{k}$ being a vector of magnitude equal to the refractive index oriented along the ray's direction of propagation.

At this point, it is expected that the skew invariant $h$ becomes the translational invariant when $c \rightarrow \infty$ and the rotational invariant when $c \rightarrow 0$. Then, we can introduce the invariant $h^{\prime}=\tanh (c) \cosh \left(u_{\min }\right) k_{v}$ in a similar way to that proposed by Miñano ${ }^{8}$ for the toroidal coordinate system. This invariant is conserved for rays propagating through the concentrator, where $c$ is a constant. Using the following change of variables,

$$
\begin{aligned}
& \lambda=\cosh (u), \\
& \mu=\cos (v),
\end{aligned}
$$

and introducing these new variables into Eq. (5), we can obtain

$$
\lambda^{2}=\frac{1}{2}\left\{\left(\frac{x^{2}+y^{2}}{c^{2}}+1\right)+\left[\left(\frac{x^{2}+y^{2}}{c^{2}}+1\right)^{2}-\frac{4 x^{2}}{c^{2}}\right]^{1 / 2}\right\},
$$




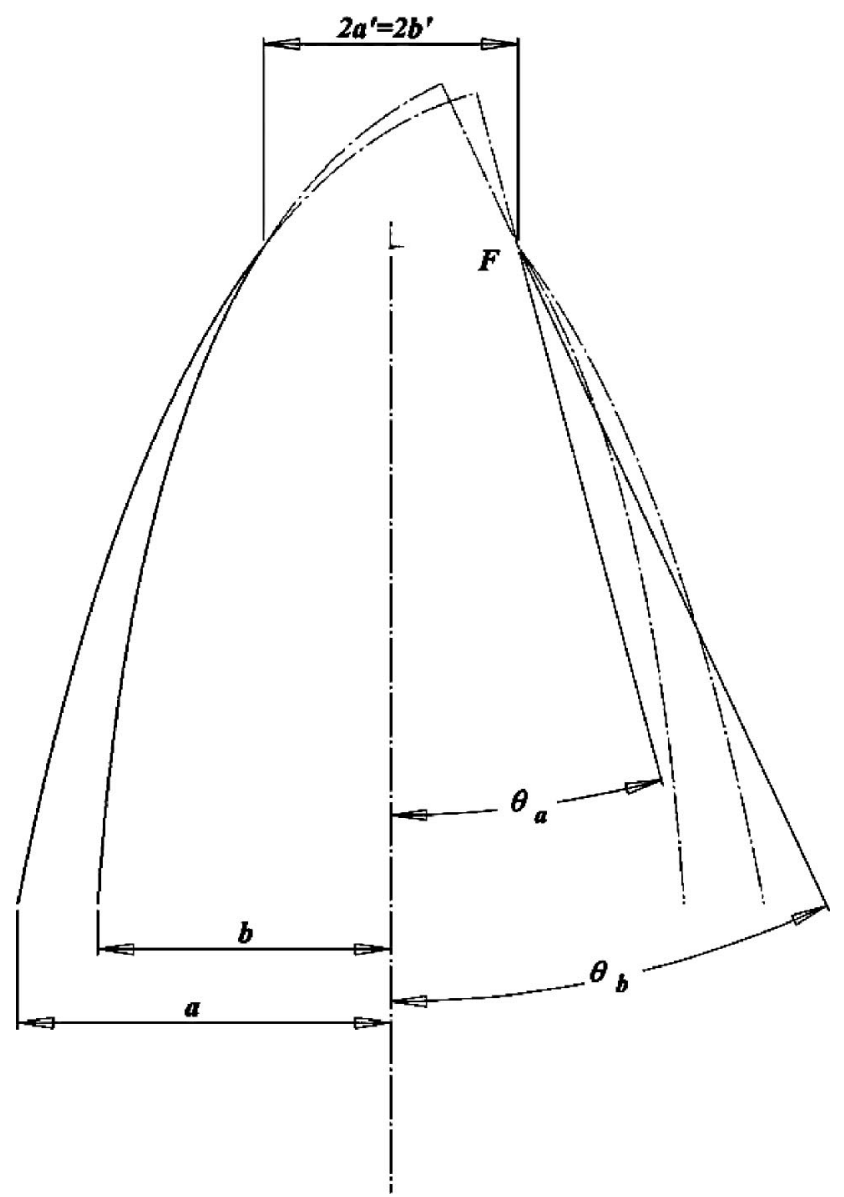

Fig. 2. Geometrical parameters of a nonhomofocal elliptical CPC with a circular exit aperture.

which relates elliptic cylindrical coordinates to Cartesian coordinates. From Eq. (8), it is possible to evaluate the limits for $h^{\prime}$, thus obtaining the translational skew invariant $\left(k_{v}\right)$ when $c \rightarrow \infty$ and the rotational skew invariant $\left(r_{\min } k_{v}\right)$ when $c \rightarrow 0$.

The invariant $h^{\prime}$ is valid for what we have called homofocal ECs, which are concentrators where all sections are ellipses with the same focal length $c$. In this sense, rotational and translational symmetric concentrators can be considered as homofocal ECs with focal length $c$ equal to 0 and $\infty$, respectively. On the other hand, it is possible to consider some interesting cases of nonhomofocal ECs that do not conserve the invariant $h^{\prime}$ and have no rotational symmetry in the elliptic cylindrical coordinate system but that can provide us with concentration ratios near the theoretical maximum of Eq. (3).

As a first case we consider a concentrator with an elliptical entrance aperture and circular exit aperture, $a>b$ and $a^{\prime}=b^{\prime}$. It is clear that this concentrator is nonhomofocal, and for this case, the relation between $\theta_{a}$ and $\theta_{b}$ is

$$
\frac{n^{\prime}}{n \sin \theta_{a}}=\frac{a}{a^{\prime}}>\frac{b}{b^{\prime}}=\frac{n^{\prime}}{n \sin \theta_{b}}
$$

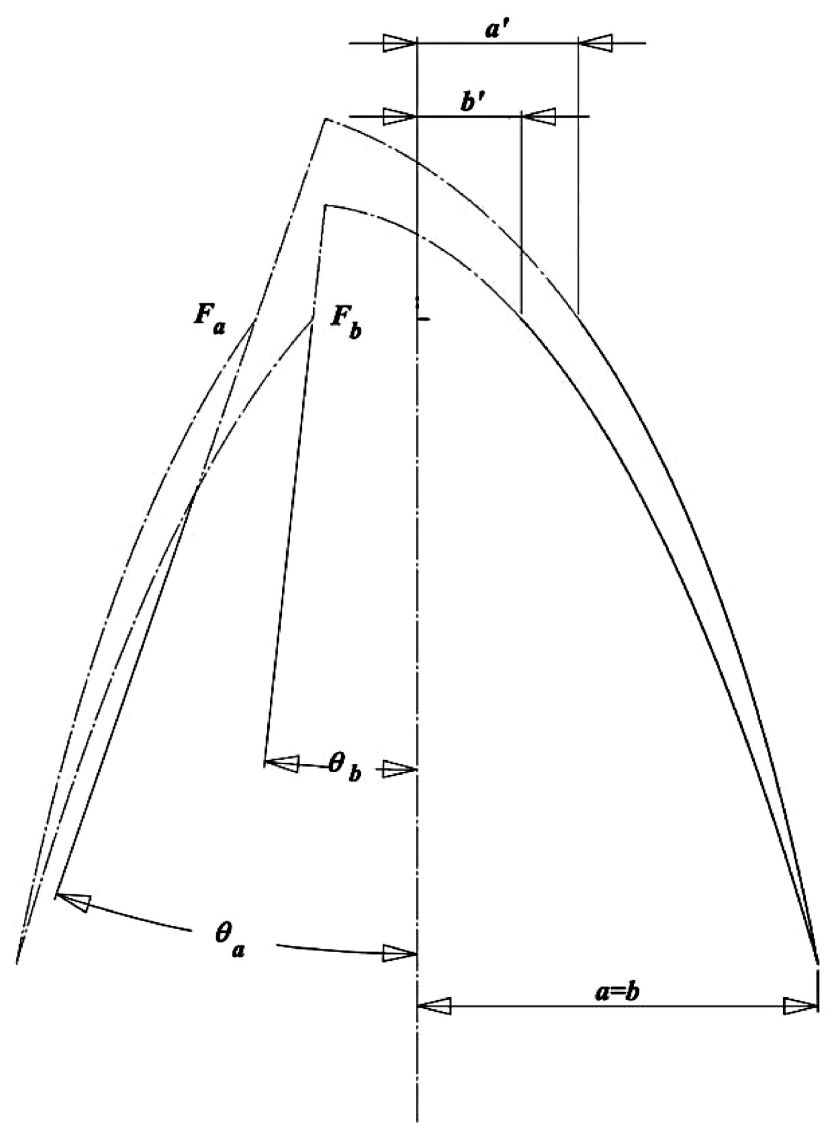

Fig. 3. Geometrical parameters of a nonhomofocal elliptical CPC with a circular entrance aperture.

which implies that $\theta_{a}<\theta_{b}$. Figure 2 shows a geometrical representation of this relation using CPCs. For two 2D CPCs with their foci, $F$, located at the same places and the same exit apertures, the one with the larger entrance aperture has the smaller acceptance angle and vice versa. In Section 4, we detail this case as an example of nonhomofocal ECs.

As a second case, we study a concentrator with an elliptical nonhomofocal entrance and exit aperture, with $a>b$ and $a^{\prime}>b^{\prime}$. In this case, the relation between $\theta_{a}$ and $\theta_{b}$ depends on the values of $a, b, a^{\prime}$, and $b^{\prime}$, and it is possible to design concentrators with $\theta_{a}>\theta_{b}, \theta_{a}=\theta_{b}$, and $\theta_{a}<\theta_{b}$.

Another nonhomofocal case occurs when $a=b$ and $a^{\prime}>b^{\prime}$ (circular entrance aperture and elliptical exit aperture),

$$
\frac{n^{\prime}}{n \sin \theta_{a}}=\frac{a}{a^{\prime}}<\frac{b}{b^{\prime}}=\frac{n^{\prime}}{n \sin \theta_{b}} ;
$$

then $\theta_{a}>\theta_{b}$. Figure 3 shows the geometrical representation for this case.

The last case corresponds to $a>b$ and $a^{\prime}<b^{\prime}$, elliptical entrance and exit apertures with crossed major axes. For this case, we have the same situation as in Eq. (8), and $\theta_{a}<\theta_{b}$. As a summary, Table 1 schematically shows the different particular cases for elliptical concentrators. 
Table 1. Relationship between $\theta_{a}$ and $\theta_{b}$ for Nonhomofocal $(\theta)$ and Homofocal $(\bigcirc)$ Concentrators

\begin{tabular}{cccccc}
\hline$a>b$ & $a>b$ & $a=b$ & $a=b$ & $a=a^{\prime}$ & $a>b$ \\
$a^{\prime}>b^{\prime}$ & $a^{\prime}=b^{\prime}$ & $a^{\prime}>b^{\prime}$ & $a^{\prime}=b^{\prime}$ & $\rightarrow \infty$ & $a^{\prime}<b^{\prime}$ \\
$(\bigcirc)$ & $(\bigcirc)$ & $(\bigcirc)$ & $(\bigcirc)$ & $(\bigcirc)$ & $(\bigcirc)$ \\
\hline- & $\theta_{a}<\theta_{b}$ & $\theta_{a}>\theta_{b}$ & $\theta_{a}=\theta_{b}$ & $\theta_{a}=\pi / 2$ & $\theta_{a}<\theta_{b}$ \\
\hline
\end{tabular}

\section{Applications of Bidirectional Elliptical Concentrators}

After the presentation of the EC, two main applications for nonimaging optical devices can be directly inferred: first as nontracking solar concentrators due to their variable angular behavior and second as optical components for illuminators with sources such as LEDs or diode lasers working in reverse mode.

It is understood that nontracking solar concentrators do not need to be guided to follow the daily movement of the Sun across the sky. Many nontracking solar concentrators have been proposed, $, 9,10$ but all of them are based on translational symmetric concentrators. As we have shown, translational symmetric concentrators can be considered ECs with a longitudinal acceptance angle equal to $\pi / 2$. However, there are applications where it is not necessary to cover the full daily movement of the Sun or where the use of secondary reflective components can be of interest. ${ }^{11}$ For these applications, it would be advantageous to employ homofocal or nonhomofocal ECs, since Eq. (4) ensures higher concentration ratios than obtained with translational concentrators, without the need for building complex and expensive tracking systems.

As a second main application, we propose the use of ECs as components in illuminators. Nonimaging concentrators have been used as optical components for LED-fiber coupling or to obtain uniform light distribution with diode lasers. ${ }^{12,13}$ In a similar way, the EC can be used for illuminators. The use of these new components can provide new capabilities, such as well-controlled astigmatic beams obtained by reflective components. Applications such as automotive or road signals, ${ }^{6,14}$ where beams with variable angles (longitudinal and transversal) are needed, are suitable applications for ECs.

\section{Numerical Results: Elliptical Compound Parabolic Concentrator}

To show the behavior of ECs, we can study the classical CPC in its elliptical configuration (ECPC). We can build homofocal ECPCs in a way completely analogous to the standard rotational symmetric CPC, rotating a $2 \mathrm{D}$ CPC profile in an elliptic cylindrical coordinate system. From a practical point of view, it could be difficult to produce this type of rotational operation, but we can obtain the same result choosing the $2 \mathrm{D}$ profile and building all sections of the concentrator as ellipses with its major or minor axes defined by the 2D CPC profile and its focal length defined by the elliptic cylindrical coordinate system parameter $c$ (Fig. 4). In this way, the equation for the ECPC in elliptic cylindrical coordinates will be the equivalent to that for the standard cylindrical coordinates,

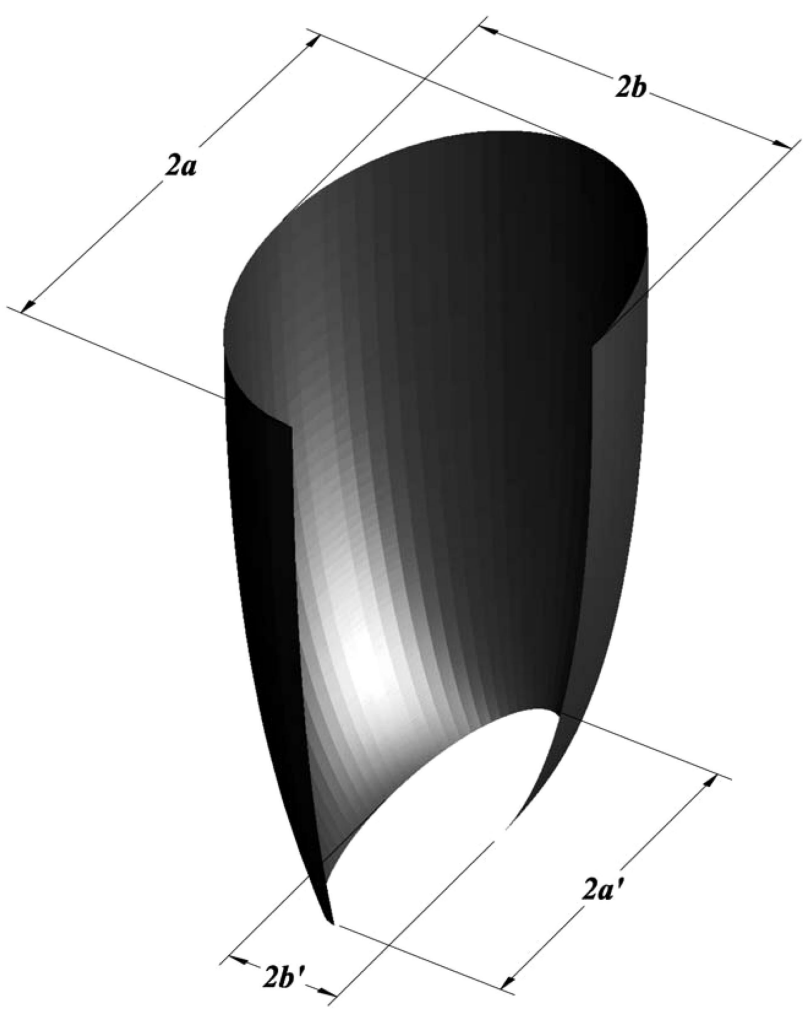

Fig. 4. Three-dimensional image for homofocal ECPC with a transversal profile of a $20^{\circ} \mathrm{CPC}$, an exit aperture of $2 b^{\prime}=20 \mathrm{~mm}$, and a focal length of $c=30 \mathrm{~mm}$.

$$
\begin{aligned}
& \left(u \cos \theta_{\max }+z \sin \theta_{\max }\right)^{2}+2 u_{0}\left(1+\sin \theta_{\max }\right)^{2} u \\
& -2 u_{0} \cos \theta_{\max }\left(2+\sin \theta_{\max }\right)^{2} z-u_{0}^{2}\left(1+\sin \theta_{\max }\right) \\
& \times\left(3+\sin \theta_{\max }\right)=0,
\end{aligned}
$$

where we have replaced the standard radius $r$ by the elliptical radius $u$, and $u_{0}$ is the elliptical radius at the exit aperture.

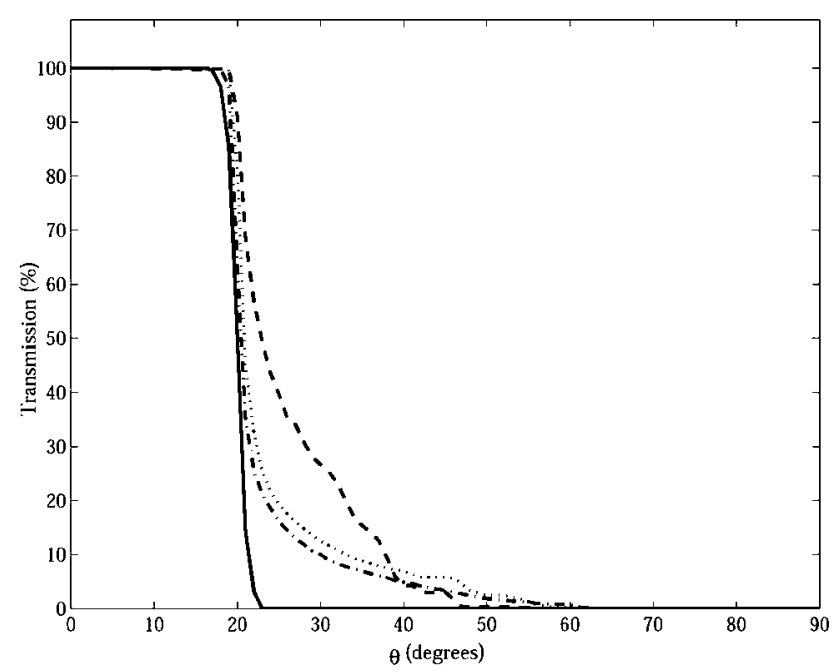

Fig. 5. Transversal transmission-angle curves for several homofocal ECPCs: (- - -), $c_{1}=99 \mathrm{~mm} ;(\cdot \cdot), c_{2}=60 \mathrm{~mm} ;(--), c_{3}=30 \mathrm{~mm}$; and $(-), c_{4}=0$ (3D CPC). 


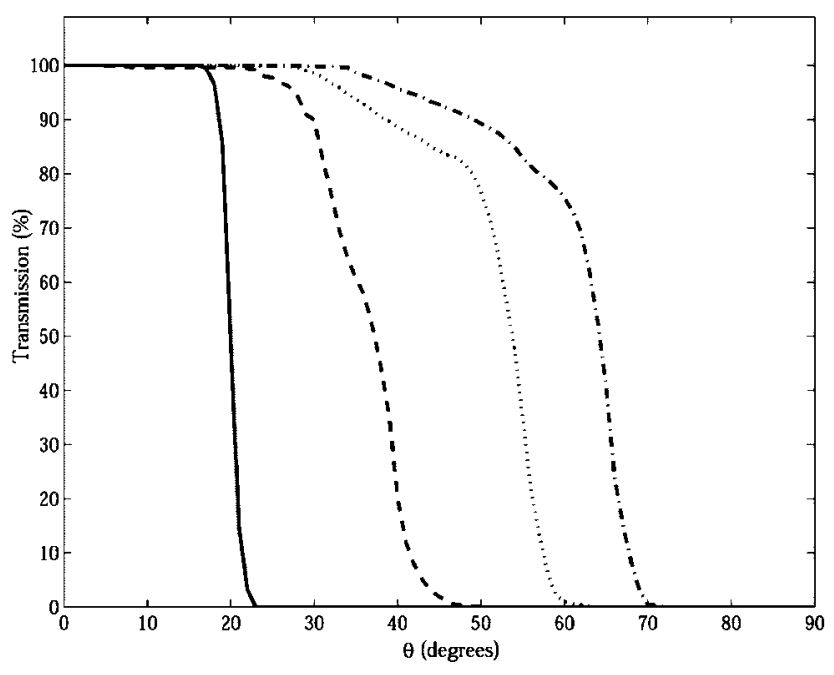

Fig. 6. Longitudinal transmission-angle curves for several homofocal ECPCs: (- -),$c_{1}=99 \mathrm{~mm} ;(\cdot), c_{2}=60 \mathrm{~mm} ;(--)$, $c_{3}=30 \mathrm{~mm}$; and (-), $c_{4}=0$ (3D CPC).

We have evaluated the transversal and longitudinal transmission for four different homofocal ECPCs by ray-tracing simulations. ${ }^{15}$ All ECPCs have, as a transversal profile, $20^{\circ} 2 \mathrm{D}$ CPCs with an exit aperture of $2 b^{\prime}=20 \mathrm{~mm}$ and different focal lengths of $c_{1}=99 \mathrm{~mm}, c_{2}=60 \mathrm{~mm}, c_{3}=30 \mathrm{~mm}$, and $c_{4}=0 \mathrm{~mm}$ (3D CPC). Using the definition $\sin \left(\theta_{\max }\right)=a^{\prime} / a^{3}$, the values of longitudinal $\theta_{\max }$ for these concentrators are $\theta_{\max -1}=74.6^{\circ}, \theta_{\max -2}=65,7^{\circ}, \theta_{\max -3}=49^{\circ}$, and $\theta_{\max -4}=20^{\circ}$. The transversal value for $\theta_{\max }$ is $20^{\circ}$ for all concentrators. Figures 5 and 6 show transversal and longitudinal transmission-angle curves, respectively. They show that it is possible to control the vari-

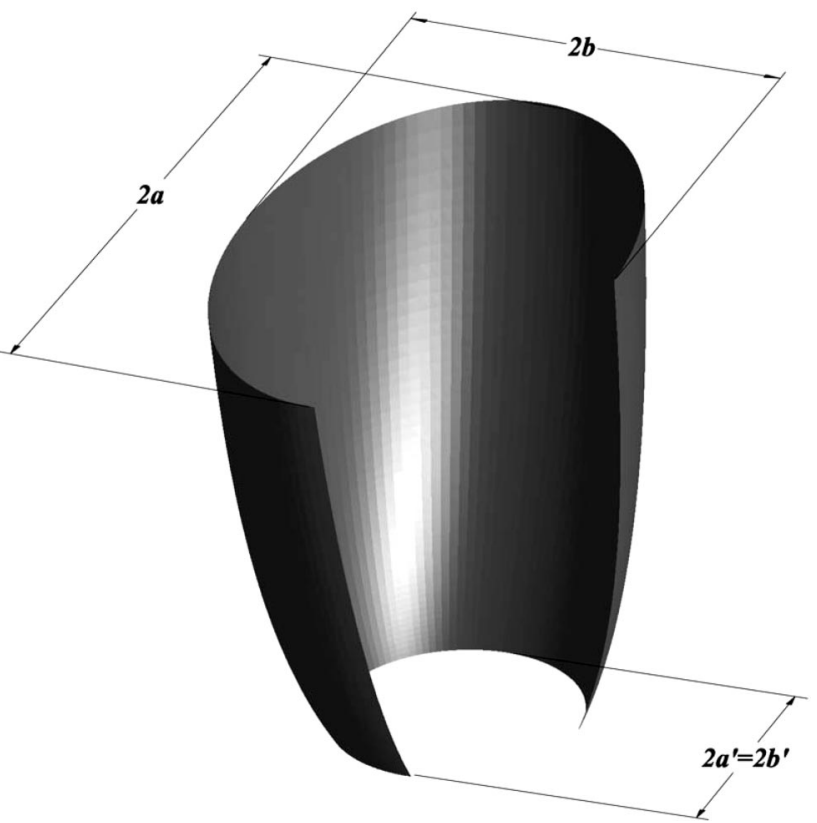

Fig. 7. Three-dimensional image for nonhomofocal ECPC with a $40^{\circ} \mathrm{CPC}$ transversal profile, a $20^{\circ} \mathrm{CPC}$ longitudinal profile, and a circular exit aperture with a radius of $10 \mathrm{~mm}$.

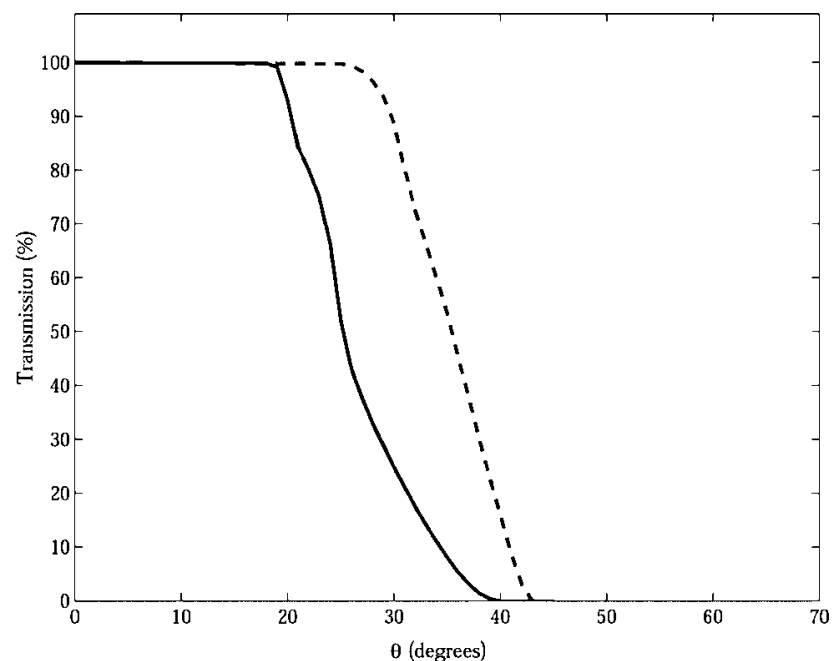

Fig. 8. Transversal (-) and longitudinal (- -) transmission-angle curve for nonhomofocal ECPCs.

able angular behavior of the concentrator with one parameter, the focal length. Small focal lengths produce a smaller angular deviation between transversal and longitudinal curves than large focal lengths do.

On the other hand, it is possible to build nonhomofocal ECPCs in a similar way to homofocal ECPCs. Instead of defining the elliptic section of the concentrator by its minor or major axes and its focal length, the concentrator can be defined by its minor axes and its major axes in such a way that its minor and major axes follow the profile of two crossed 2D CPCs. Figure 7 shows a nonhomofocal ECPC with a $40^{\circ} \mathrm{CPC}$ as the minor axis (transversal) profile and a $20^{\circ} \mathrm{CPC}$ as the major axis (longitudinal) profile, with a circular exit aperture radius of $10 \mathrm{~mm}$. The concentrator has been truncated in its longitudinal axes, to avoid the different height of the CPC for the two axes, in a way that longitudinal and transversal profiles have the same height. This results in their maximum theoretical acceptance angles being $\theta_{\max -T}=40^{\circ}$ and $\theta_{\max -L}=$ $24.1^{\circ}$. Figure 8 shows the transmission-angle curve for this nonhomofocal ECPC in longitudinal and transversal directions. It is possible to see that the nonhomofocal ECPC fits the theoretical longitudinal $\theta_{\max }$ better than the homofocal ECPC, but both are worse than the ideal EC.

\section{Conclusions}

In this paper, we have presented what we believe is a new family of nonimaging concentrators-elliptical concentrators-with interesting properties. ECs have two principal acceptance angles in the transversal and longitudinal directions. Their maximum theoretical concentration ratio is greater than the concentration ratio for translational symmetric concentrators and smaller than for rotational ones. Translational and rotational concentrators can be considered as particular cases of this new family of concentrators. ECs have their properties of symmetry well defined in an elliptic cylindrical coordinate system, such as rotational sym- 
metry or skew invariant. Based on these properties of symmetry, it is possible to define two classes of EC, homofocal and nonhomofocal. We have studied a particular case of EC-the elliptical compound parabolic concentrator-in its two configurations, homofocal and nonhomofocal. The homofocal configuration allows us to control the variable angular behavior of the concentrator by means of one parameter, the focal length of the elliptic cylindrical coordinate system. Nevertheless, new geometrical parameters and configurations are being investigated to improve the efficiency of the EC.

This research was partially supported by the project Architectural Sensors-Communidad de Madrid Programme ref. S-505/ENE0355. The authors thank Agustin Gonzalez-Cano for his contribution to this work.

\section{References}

1. H. Hintenberger and R. Winston, "Efficient light coupler for threshold Cernkov counters," Rev. Sci. Instrum. 30, 10941095 (1966).

2. R. Winston, "Light collection within the framework of geometrical optics," J. Opt. Soc. Am. 60, 245-247 (1970).

3. R. Winston, J. C. Miñano, and P. Benitez, with contributions by N. Shatz and J. C. Bortz, Nonimaging Optics (Elsevier 2005).

4. D. Jenkins, R. Winston, J. Bliss, J. O'Gallagher, A. Lewandowski, and C. Bingham, "Solar concentration of 50,000 achieved with output power approaching $1 \mathrm{~kW}$," J. Sol. Energy Eng. 118, 141-145 (1996).

5. P. T. Ong, J. M. Gordon, and A. Rabl, "Tailoring lighting reflectors to prescribed illuminance distributions: compact partial-involute designs,” Appl. Opt. 34, 7877-7887 (1995).

6. P. Benítez, J. C. Miñano, J. Blen, R. Mohedano, J. Chaves, O. Cross, M. Hernández, and W. Falicoff, "Simultaneous multiple surface optical design method in three dimensions," Opt. Eng. 43, 1489-1502 (2004).

7. H. A. Buchdahl, An Introduction to Hamiltonian Optics (Dover, 1993).

8. J. C. Miñano, "Cylindrical concentrators as a limit case of toroidal concentrators," Appl. Opt. 23, 2017-2020 (1984).

9. W. Spirkl, H. Ries, J. Muschaweck, and R. Winston, "Nontracking solar concentrators," Sol. Energy 62, 113-120 (1998).

10. C. J. Sletten, F. S. Holt, and S. B. Herskovitz, "Wide-angle lenses and image collapsing subreflectors for nontracking solar collectors," Appl. Opt. 19, 1439-1453 (1980).

11. H. Ries, J. M. Gordon, and M. Laxen, "High-flux photovoltaic solar concentrator with kaleidoscope-based optical design," Sol. Energy 60, 11-16 (1997).

12. C. Rooman, M. Kuijk, R. Vounckx, and P. Heremans, "Reflective-refractive microlens for efficient light emitting diode to fiber coupling," Opt. Eng. 44, 095005 (2005).

13. T. Alahautala and R. Hernberg, "Generation of uniform light by use of diode lasers and a truncated paraboloid with a Lambertian scatterer," Appl. Opt. 43, 949-954 (2004).

14. A. García-Botella, Ph.D. dissertation (Universidad Complutense de Madrid, 2000).

15. TracePro software, http://www.lambdares.com/. 